\title{
Estudio comparativo entre banda de tensión y tornillo compresivo para la artrodesis interfalángica proximal y metacarpofalángica
}

\author{
Javier Bennice, Gerardo L. Gallucci, Ezequiel E. Zaidenberg, Pablo De Carli, Jorge G. Boretto \\ Sector de Cirugía de Mano y Miembro Superior, Instituto de Ortopedia y Traumatología "Prof. Dr. Carlos E. Ottolenghi", \\ Hospital Italiano de Buenos Aires, Ciudad Autónoma de Buenos Aires, Argentina
}

\begin{abstract}
RESUMEN
Introducción: Los métodos más utilizados para la artrodesis de la articulación interfalángica proximal y metacarpofalángica son la banda de tensión y el tornillo compresivo. El objetivo de este estudio fue comparar los resultados entre pacientes tratados con estas técnicas. Materiales y Métodos: Estudio comparativo retrospectivo de 10 años. Se incluyeron pacientes esqueléticamente maduros tratados por artrosis y artritis. Se analizaron la tasa de consolidación ósea, el tiempo hasta la consolidación, la incidencia de complicaciones y la tasa de reoperaciones. En un análisis secundario, se compararon los pacientes reumáticos y no reumáticos. Resultados: Se evaluaron 56 casos en 44 pacientes (edad promedio 53 años). Grupo 1: 35 casos tratados con banda de tensión y grupo 2: 21 tratados con tornillo compresivo. Había 32 casos reumáticos y 24 no reumáticos. El seguimiento promedio fue de 24 meses. La tasa promedio de consolidación fue del $94,2 \%$ en el grupo 1 y del $85,7 \%$ en el grupo 2 . La incidencia de complicaciones fue del $11,4 \%$ en el primer grupo y $23,8 \%$ en el segundo, y la tasa de reoperaciones, del $17,1 \%$ y del $0 \%$, respectivamente. Conclusiones: Ambos métodos permiten lograr tasas altas de consolidación, pero la incidencia de seudoartrosis fue casi tres veces superior con el tornillo compresivo que con la banda de tensión. La tasa más alta de reoperaciones en el grupo con banda de tensión fue por extracción del material. El tiempo de consolidación fue más corto en los pacientes no reumáticos, independientemente de la técnica.
\end{abstract}

Palabras clave: Artrodesis; fusión interfalángica proximal y metacarpofalángica; banda de tensión; tornillo compresivo.

Nivel de Evidencia: III

Comparative study between tension band and compression screw fixation in metacarpophalangeal and proximal interphalangeal joint arthrodesis

\begin{abstract}
Introduction: Tension band wiring (TBW) and compression screw fixation are the most common methods used for proximal interphalangeal (PIP) and metacarpophalangeal (MCP) joint arthrodesis. The aim of this study was to compare outcomes between patients treated with those methods. Materials and Methods: A 10-year retrospective comparative study. The study population included skeletally mature patients treated for osteoarthritis or arthritis. Union rates, healing times, complications, and reoperation rates were compared between TBW and compression screw fixation methods. Outcomes were also studied in terms of rheumatic and nonrheumatic patients. Results: The study sample consisted of 56 cases and 44 patients (average age, 53 years). Group 1 : 35 patients treated with TBW. Group 2: 21 patients treated with compression screw fixation. There were 32 rheumatic cases and 24 nonrheumatic cases. The average follow-up was 24 months. Union rates were 94.2\% (Group 1) and 85.7\% (Group 2). Complication rates were $11.4 \%$ (Group 1) and 23.8\% (Group 2). Reoperation rates were 17.1\% (Group 1) and 0\% (Group 2). Conclusions: Both methods have high union rates; however, the nonunion incidence in the compression screw group was almost three times higher than in the TBW group. The reoperation rate was higher in the TBW group, mostly due to hardware removal. Healing time was shorter in non-rheumatic patients regardless of the method.
\end{abstract}

Key words: Arthrodesis; proximal interphalangeal and metacarpophalangeal joint fusion; tension band; compression screw. Level of Evidence: III

Recibido el 27-2-2020. Aceptado luego de la evaluación el 6-6-2020 • Dr. JAVIER BENNICE • bennicejavier@gmail.com ID https://orcid.org/0000-0001-6004-1240 Cómo citar este artículo: Bennice J, Gallucci GL, Zaidenberg EE, De Carli P, Boretto JG. Estudio comparativo entre banda de tensión y tornillo compresivo para la artrodesis interfalángica proximal y metacarpofalángica. Rev Asoc Argent Ortop Traumatol 2020;85(4):325-334. https://doi.org/ 10.15417/issn. 1852-7434.2020.85.4.1063 


\section{INTRODUCCIÓN}

Varias situaciones clínicas pueden provocar dolor intenso, deformidad o inestabilidad de las articulaciones de la mano, algunas de las causas más frecuentes son la artrosis primaria y secundaria, y las enfermedades inflamatorias, como la artritis reumatoide. Aunque uno de los objetivos fundamentales del cirujano ortopédico es preservar la movilidad de las articulaciones de la mano, cuando fracasa el tratamiento conservador se deben tener en cuenta y ofrecer al paciente las opciones quirúrgicas, entre ellas, uno de las más difundidas es la artrodesis. ${ }^{1-3}$

La artrodesis sigue siendo el patrón de referencia para aliviar el dolor y obtener estabilidad. ${ }^{4}$ El objetivo de la fusión articular es lograr un contacto y una compresión adecuados entre las superficies óseas para conseguir la consolidación. Una fijación estable permite una movilización temprana de las articulaciones adyacentes y así evitar la rigidez articular. ${ }^{5}$

Existen diferentes métodos para la fijación ósea y los más utilizados son la banda de tensión y el tornillo compresivo. ${ }^{6-8}$ Se ha demostrado que ambas técnicas logran una compresión y una estabilidad adecuadas, con buenos resultados clínicos y radiográficos. ${ }^{9-18}$ La banda de tensión tiene como ventaja su bajo costo, aunque se reportan tasas más altas de extracción del material debido a las molestias del implante, ${ }^{7,-13}$ mientras que el tornillo compresivo es más caro, pero la tasa de retiro del material es más baja. ${ }^{8,14-18}$

Se han llevado a cabo estudios biomecánicos que comparan la rigidez de los diferentes métodos de fijación articular, ${ }^{19-23}$ pero se han publicado pocos estudios clínicos comparativos entre las diferentes técnicas para la fusión articular en la mano. ${ }^{5}$ Incluso algunos estudios comparan técnicas de artrodesis que, según ya se demostró, ofrecen resultados menos satisfactorios, como las clavijas de Kirschner. ${ }^{24,25}$

El objetivo de este estudio fue comparar los resultados de la artrodesis interfalángica proximal y metacarpofalángica en pacientes tratados con técnica de banda de tensión o con tornillo compresivo.

\section{MATERIALES Y MÉTODOS}

Se llevó a cabo un estudio de cohortes comparativo retrospectivo entre banda de tensión y tornillo compresivo para la artrodesis interfalángica proximal y metacarpofalángica, en un período de 10 años. Como criterio de inclusión se consideraron los pacientes esqueléticamente maduros tratados por artrosis primaria, secundaria o artritis, a quienes se les realizó una fijación articular interfalángica proximal o metacarpofalángica con alguno de estos dos métodos. Se excluyó a los pacientes que no tuvieron un seguimiento clínico o radiográfico en el posoperatorio. Todas las cirugías fueron realizadas o supervisadas directamente por cirujanos de mano experimentados. La selección del método para la artrodesis no fue aleatoria, sino que estuvo determinada por la preferencia de cada cirujano, en cada caso en particular.

Los datos se recolectaron de la historia clínica electrónica. Se analizaron las variables demográficas, la causa de la degeneración articular, las articulaciones y los dedos involucrados, y el método utilizado para la fijación articular. Todos los pacientes fueron evaluados clínicamente y con radiografías en el posoperatorio. Se compararon la tasa de consolidación ósea, el tiempo hasta la consolidación, la incidencia de complicaciones y la tasa de reoperaciones.

Además del análisis comparativo entre el grupo tratado con banda de tensión y el grupo tratado con tornillo compresivo, también se compararon los resultados entre los pacientes reumáticos y no reumáticos, al margen del método de artrodesis utilizado en cada uno de ellos.

Con respecto a la técnica quirúrgica, las artrodesis se realizaron a partir de un abordaje dorsal. Se resecó el cartílago articular de ambas superficies óseas y se lo preparó mediante el método cóncavo-convexo. En el grupo con banda de tensión, la fijación se hizo mediante dos clavijas de Kirschner paralelas y alambre con configuración en ocho. En el otro grupo, se utilizaron tornillos autocompresivos canulados con técnica anterógrada. Se colocaron uno o dos tornillos de acuerdo con la necesidad de cada caso en particular.

Siempre se prestó mucha atención a obtener una correcta rotación del dedo y lograr un contacto y una compresión adecuados entre las superficies óseas.

En la evaluación posoperatoria, todos los pacientes fueron citados para controles periódicos clínicos y radiográficos. Se tomaron radiografías de frente y de perfil estrictas del dedo involucrado hasta obtener la consolidación ósea de la fijación articular.

Retrospectivamente un autor independiente que no estuvo involucrado en el tratamiento quirúrgico de los pacientes evaluó las radiografías posoperatorias para determinar la consolidación ósea y el tiempo hasta la consolidación de la artrodesis. La consolidación ósea fue definida como la presencia de trabéculas óseas a través del sitio de la fijación en más del 50\% de esta área. Se consideró retraso de la consolidación a aquellas 
consolidaciones óseas que se produjeron a las 12 semanas o más de la cirugía. La aparición de complicaciones en el posoperatorio y la necesidad de reoperaciones se evaluaron mediante la recolección de datos de la historia clínica electrónica.

\section{Análisis estadístico}

Se llevó a cabo un análisis estadístico descriptivo. Las variables categóricas se informan como valores absolutos o relativos (porcentajes). Para las variables continuas se informan las medidas de tendencia central como media y las medidas de dispersión como rango y desviación estándar.

\section{RESULTADOS}

En un período de 10 años, desde agosto de 2008 hasta agosto de 2018, se identificaron 60 casos en 47 pacientes que cumplieron con los criterios de inclusión. Finalmente se evaluaron 56 casos en 44 pacientes ( 22 hombres y 22 mujeres, edad promedio 53 años [rango 17-79]), ya que cuatro se perdieron en el seguimiento. En 35 casos $(62,5 \%)$, se había utilizado la banda de tensión como método de artrodesis y, en 21 (37,5\%), la fijación con tornillo compresivo. El seguimiento posoperatorio promedio era de 24 meses (rango 6-100). La indicación de la artrodesis había sido por enfermedad reumática (32 casos, $57 \%$ ) y por causas no reumáticas ( 24 casos, $43 \%$ ). Entre los tratados por enfermedad reumática, 26 casos correspondían a artritis reumatoide; tres, a artritis psoriásica; dos, a artritis idiopática juvenil y uno, a enfermedad de Still. Las indicaciones en los pacientes no reumáticos fueron: artrosis primaria ( 2 casos), artrosis postraumática (11 casos), inestabilidad crónica (6 casos) y otras causas en los cinco restantes (artrodesis de acortamiento en la enfermedad de Dupuytren [1 caso], secuela de lesión del tendón flexor [1 caso], parálisis cubital [1 caso] y mano espástica con pulgar incluido [2 casos]). En 18 casos, la artrodesis era interfalángica proximal $(32 \%)$ y, en 38 casos, metacarpofalángica (68\%). Las artrodesis interfalángicas proximales fueron: dedo índice ( 2 casos), dedo mayor ( 2 casos), dedo anular (4 casos) y meñique (10 casos). Todas las fijaciones metacarpofalángicas se hicieron en el pulgar, salvo un caso en el meñique. Los dedos involucrados en las artrodesis fueron: pulgar (37 casos, 66\%), índice ( 2 casos, 3,5\%), mayor ( 2 casos, $3,5 \%$ ), anular (4 casos, $7 \%$ ) y meñique (11 casos, 20\%) (Tabla 1 ).

Se obtuvo la consolidación ósea en 33 de 35 casos $(94,2 \%)$ del grupo con banda de tensión, en un promedio de 6.4 semanas (rango 4-12) (Figura 1) y en 18 de 21 casos (85,7\%) del grupo con tornillo compresivo, en un promedio de 7.2 semanas (rango 4-15) (Figura 2). Cuatro de los 35 casos $(11,4 \%)$ con banda de tensión y cinco de los 21 casos $(23,8 \%)$ con tornillo compresivo tuvieron complicaciones. En el grupo con banda de tensión, seis de 35 casos $(17,1 \%)$ requirieron una reoperación, mientras que ningún paciente del grupo con tornillo compresivo fue operado nuevamente $(0 \%)$ (Tabla 2$)$.

Por otro lado, se logró la consolidación ósea en 29 de 32 pacientes reumáticos $(90,6 \%)$, en un promedio de 7.2 semanas (rango 4-15) y en 22 de 24 casos no reumáticos (91,6\%), en un promedio de 6 semanas (rango 4-12). Seis de 32 pacientes $(18,7 \%)$ del grupo de reumáticos y tres de $24(12,5 \%)$ del grupo de no reumáticos tuvieron complicaciones. Dos de 32 pacientes reumáticos $(6,2 \%)$ y cuatro de 24 no reumáticos $(16,6 \%)$ fueron operados nuevamente (Tabla 3).

Con respecto a las complicaciones, en el grupo con banda de tensión, hubo dos casos de seudoartrosis, uno de retraso de la consolidación ósea y uno de ruptura del extensor largo del pulgar. Uno de los casos de seudoartrosis ocurrió en un paciente no reumático en el que se realizó la revisión de la artrodesis a partir de la conversión a una fijación con placa de osteosíntesis con autoinjerto óseo y finalmente se logró la consolidación. El otro caso fue en un paciente reumático, pero la seudoartrosis era asintomática y no fue necesaria una nueva intervención quirúrgica. Ambas seudoartrosis eran atróficas. Un paciente no reumático tuvo un retraso de la consolidación (a las 12 semanas de la cirugía). Un paciente reumático sufrió una ruptura tendinosa a los 6 meses de la intervención que fue resuelta satisfactoriamente con la extracción del material de osteosíntesis y la transferencia tendinosa del extensor propio del índice.

En el grupo con tornillo compresivo, hubo tres casos de seudoartrosis y dos de retraso de la consolidación ósea. Un paciente no reumático tuvo seudoartrosis, pero se negó a someterse a una cirugía de revisión, mientras que los otros dos casos ocurrieron en pacientes reumáticos y fueron asintomáticos, por lo que no requirieron de una nueva cirugía. Todas las seudoartrosis eran atróficas (Figura 3). Ambos retrasos de la consolidación ocurrieron en pacientes reumáticos y la consolidación ósea se produjo a las 14 y a las 15 semanas de la cirugía. No se registraron otras complicaciones, como infecciones ni fracturas intraoperatorias. 
Tabla 1. Datos demográficos

\begin{tabular}{|c|c|c|}
\hline Variables & Banda de tensión & Tornillo compresivo \\
\hline Número de casos & 35 & 21 \\
\hline Edad, años (rango) & $52.8(17-79)$ & $54.1(24-69)$ \\
\hline Hombres (\%) & $18(58,0)$ & $4(28,5)$ \\
\hline Reumáticos (\%) & $14(40,0)$ & $18(85,7)$ \\
\hline Seguimiento, meses (rango) & $28.1(6-100)$ & $18.3(6-43)$ \\
\hline \multicolumn{3}{|l|}{ Articulaciones } \\
\hline Interfalángica proximal & 13 & 5 \\
\hline Metacarpofalángica & 22 & 16 \\
\hline \multicolumn{3}{|l|}{ Dedos } \\
\hline Pulgar & 22 & 15 \\
\hline Índice & 1 & 1 \\
\hline Mayor & 1 & 1 \\
\hline Anular & 3 & 1 \\
\hline Meñique & 8 & 3 \\
\hline \multicolumn{3}{|l|}{ Indicación } \\
\hline Artrosis primaria & 2 & 0 \\
\hline Artrosis postraumática & 11 & 0 \\
\hline Inestabilidad crónica & 5 & 1 \\
\hline Otras causas & 3 & 2 \\
\hline Artritis reumatoide & 8 & 18 \\
\hline Artritis psoriásica & 3 & 0 \\
\hline Artritis idiopática juvenil & 2 & 0 \\
\hline Enfermedad de Still & 1 & 0 \\
\hline
\end{tabular}

En cuanto a las reoperaciones, todas se realizaron en pacientes con banda de tensión, fueron seis casos ( 2 en pacientes reumáticos y 4 en no reumáticos). A cuatro pacientes se les extrajo la osteosíntesis por intolerancia al material. Las restantes dos reoperaciones se efectuaron para la revisión de la artrodesis por seudoartrosis y para la transferencia tendinosa por ruptura de un tendón, respectivamente.

\section{DISCUSIÓN}

La artrodesis interfalángica proximal y metacarpofalángica se utiliza ampliamente para mejorar la función de la mano en pacientes con artrosis primaria y secundaria severa o con enfermedad reumática, pues permite eliminar el dolor y la inestabilidad articular, y posicionar los dedos en una actitud funcional. Los métodos de fijación han sufrido cambios en las últimas décadas, ya que la aparición de nuevas tecnologías e investigaciones ha permitido el desarrollo de implantes que ofrecen una fijación más segura y la posibilidad de una movilización temprana de las articulaciones adyacentes, pero, al mismo tiempo, este avance ha generado nuevas complicaciones y retos técnicos para tener en cuenta. ${ }^{26}$ 


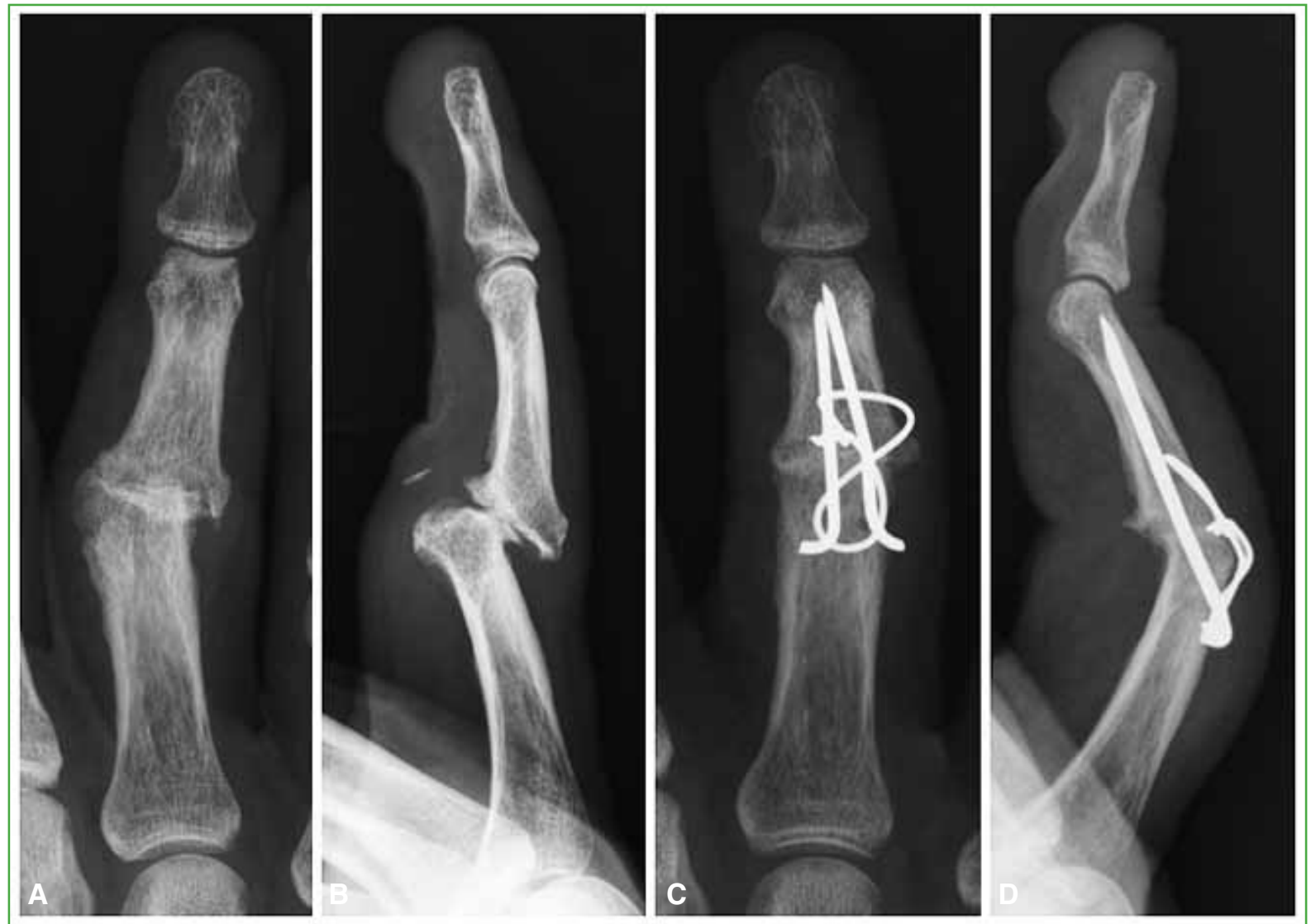

Figura 1. A y B. Artrosis interfalángica proximal de anular, postraumática en un hombre de 63 años. C y D. Artrodesis con banda de tensión, control a los 6 meses de la cirugía.

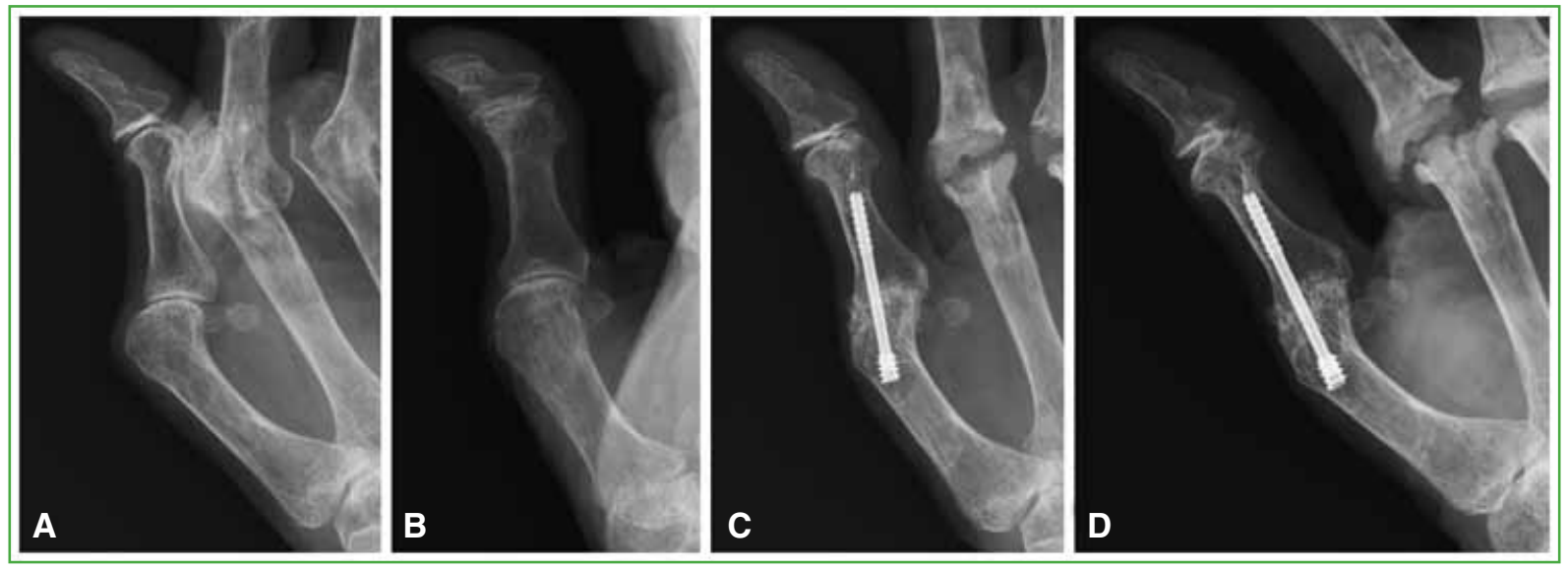

Figura 2. A y B. Compromiso de la articulación metacarpofalángica del pulgar por artritis reumatoide en una mujer de 61 años. C y D. Artrodesis con tornillo compresivo, control al año de la cirugía. 
Tabla 2. Resultados comparativos entre las técnicas quirúrgicas

Variables Banda de tensión

Tasa de consolidación

$\mathrm{n} / \mathrm{total}(\%)$

$33 / 35(94,2)$

$18 / 21(85,7)$

Tiempo de consolidación

Tiempo en semanas (DE)

$6.4(2.0)$

$7.2(2.7)$

Tasa de complicaciones

Pacientes (\%)

4/35 $(11,4)$

$5 / 21(23,8)$

Seudoartrosis (\%)

$2 / 35(5,7)$

$3 / 21(14,2)$

Retraso de la consolidación (\%)

$1 / 35(2,8)$

$2 / 21(9,5)$

Ruptura tendinosa (\%)

$1 / 35(2,8)$

$0 / 21(0)$

Tasa de reoperaciones

Pacientes (\%)

$6 / 35(17,1)$

$0 / 21(0)$

Extracción del material (\%)

$4 / 35(11,4)$

$0 / 21(0)$

Revisión de la artrodesis (\%)

$1 / 35(2,8)$

$0 / 21(0)$

Transferencia tendinosa (\%)

$1 / 35(2,8)$

$0 / 21(0)$

DE = desviación estándar

Tabla 3. Resultados comparativos entre pacientes reumáticos y no reumáticos

\section{Variables}

Tasa de consolidación

$\mathrm{n} / \mathrm{total}(\%)$

Tiempo de consolidación

Tiempo en semanas (DE)

Tasa de complicaciones

$$
\text { Pacientes }(\%)
$$

Seudoartrosis $(\%)$

Retraso de la consolidación (\%)

Ruptura tendinosa (\%)

Tasa de reoperaciones

Pacientes (\%)

Extracción del material (\%)

Revisión de la artrodesis (\%)

Transferencia tendinosa (\%)

$\mathrm{DE}=$ desviación estándar
$29 / 32(90,6)$

Pacientes reumáticos

Pacientes no reumáticos

$22 / 24(91,6)$

$7.2(2.3)$

$6.0(2.1)$

$3 / 24(12,5)$

$2 / 24(8,3)$

$3 / 32(9,3)$

$1 / 24(4,1)$

$2 / 32(6,2)$

0/24 (0)

$1 / 32(3,1)$

$2 / 32(6,2)$

$4 / 24(16,6)$

$1 / 32(3,1)$

$3 / 24(12,5)$

0/32 (0)

$1 / 24(4,1)$

$1 / 32(3,1)$ $0 / 24(0)$ 


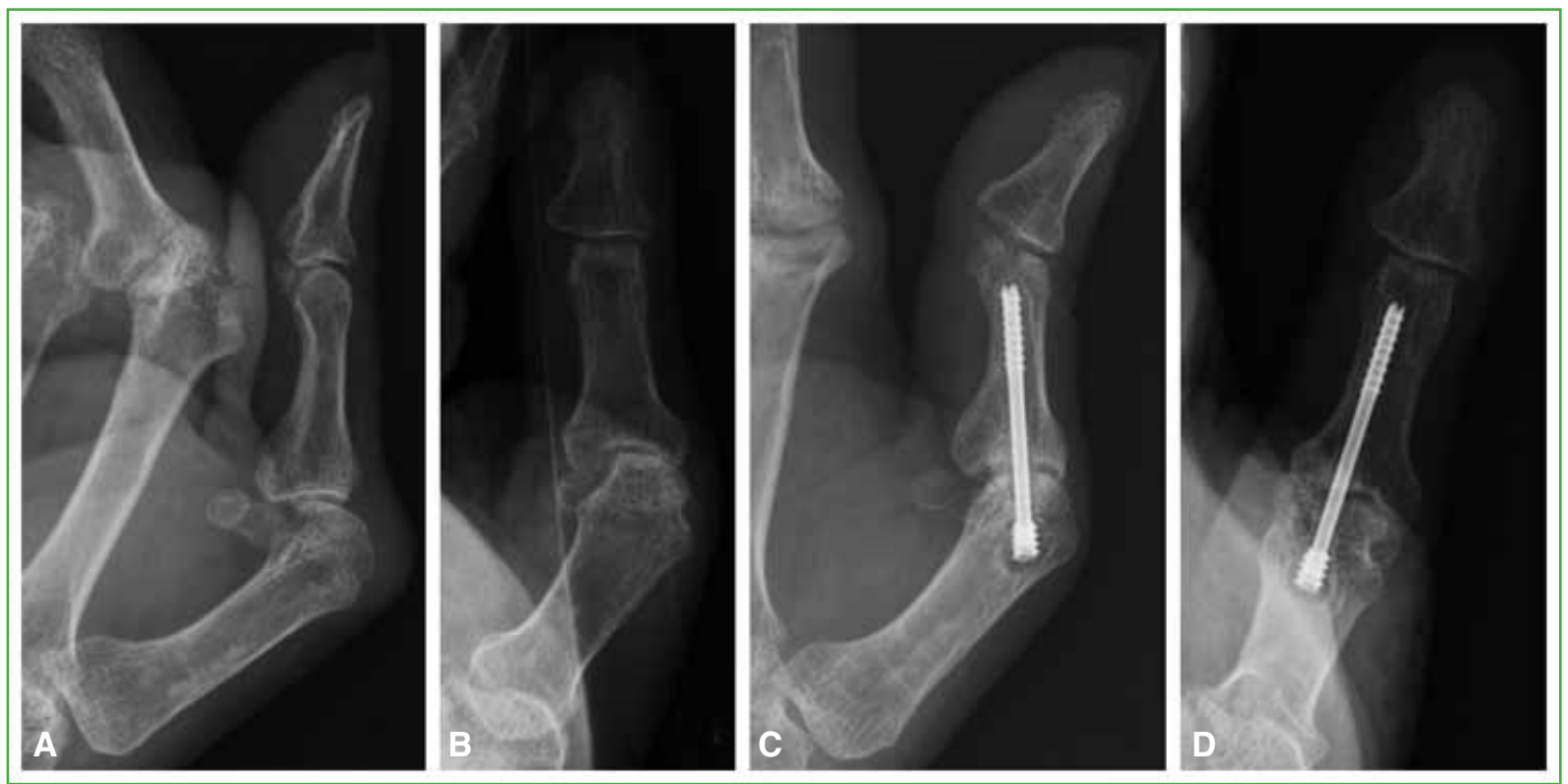

Figura 3. A y B. Compromiso de la articulación metacarpofalángica del pulgar por artritis reumatoide en una mujer de 59 años. C y D. Seudoartrosis atrófica de la fijación con tornillo compresivo, control a los 18 meses de la cirugía.

La banda de tensión y el tornillo compresivo son los métodos más utilizados para la fijación articular y se ha demostrado que logran una compresión y una estabilidad adecuadas, con buenos resultados clínicos y radiográficos. Las ventajas de la banda de tensión son el menor costo, la mayor accesibilidad y que la compresión entre las superficies óseas en el sitio de la fijación se produce durante la movilidad activa, pero con este método, se comunican tasas más altas de extracción del material por molestias del implante. ${ }^{7,9-13}$ Por otro lado, el tornillo compresivo tiene una menor tasa de retiro del material y, de acuerdo con algunos estudios, la tasa de seudoartrosis sería más baja ${ }^{25}$ sus desventajas son el costo más alto y cierta limitación para lograr el ángulo de fijación deseado, porque, si bien el desarrollo del tornillo canulado ha facilitado la técnica de colocación, el grado de flexión que permite este método continúa siendo más limitado que el que ofrece la banda de tensión. ${ }^{26}$

Se han publicado múltiples estudios que comunican buenos resultados de la artrodesis con banda de tensión. Stern y cols. ${ }^{12}$ publicaron una serie de 290 artrodesis en 203 pacientes con banda de tensión para las articulaciones interfalángica proximal y metacarpofalángica, con una tasa de consolidación ósea del $97 \%$ y una tasa de extracción del material del 9\%. En un estudio retrospectivo de 76 fijaciones articulares con banda de tensión en 63 pacientes, Uhl y Schneider ${ }^{13}$ obtuvieron la consolidación ósea en el 99\% de los casos y 10 pacientes requirieron el retiro del material. Khuri ${ }^{11}$ reportó la consolidación ósea en todos los casos de una serie de artrodesis con banda de tensión en cinco articulaciones metacarpofalángicas y 10 interfalángicas proximales; en cuatro casos, fue necesario extraer el material de osteosíntesis. Por último, en un estudio reciente de Hohendorff y cols., ${ }^{10}$ se evaluó a 15 pacientes con artrodesis interfalángica proximal con banda de tensión. Se obtuvo la consolidación ósea y los pacientes estaban muy satisfechos con el resultado del procedimiento y sus actividades de la vida diaria no estaban afectadas.

Por otro lado, distintas publicaciones comunican resultados satisfactorios con el tornillo compresivo como método de fijación articular en la mano. Ayres y cols. ${ }^{15}$ informan una tasa de consolidación del $98 \%$ en una serie de 51 artrodesis interfalángicas proximales con tornillo compresivo y una tasa de extracción del material del $4 \%$. Katzman y cols. ${ }^{16}$ también lograron buenos resultados en una serie de 51 artrodesis interfalángicas con tornillo compresivo; obtuvieron la consolidación ósea en todos los casos, sin necesidad de extraer el material en ningún paciente. Asimismo, Teoh y cols. ${ }^{18}$ comunicaron una tasa de consolidación del $96 \%$ sin casos de retiro del material en 23 artrodesis interfalángicas con tornillo compresivo, nueve casos correspondían a la articulación interfalángica proximal. 
Sin embargo, pese a la amplia difusión de la artrodesis como recurso de rescate en la cirugía de la mano, son muy escasas las publicaciones de estudios clínicos comparativos entre los diferentes métodos de fusión articular. En un estudio retrospectivo, Ijsselstein y cols. ${ }^{24}$ compararon los resultados de la fijación con clavijas de Kirschner y con banda de tensión en las artrodesis metacarpofalángica e interfalángica y comunicaron tasas de infección y de segunda artrodesis significativamente más altas en los pacientes tratados solo con clavijas. Leibovic y Strickland ${ }^{25}$ publicaron un análisis comparativo retrospectivo de múltiples métodos para la artrodesis interfalángica proximal en 224 casos. La tasa de seudoartrosis más baja se obtuvo en la fijación con tornillo de Herbert; la tasa intermedia, con banda de tensión y la más alta, con clavijas de Kirschner. Por último, Breyer y cols. ${ }^{5}$ realizaron un estudio comparativo retrospectivo entre banda de tensión y tornillo compresivo para la artrodesis metacarpofalángica, interfalángica proximal e interfalángica del pulgar, con resultados similares entre ambos métodos en cuanto a la consolidación ósea, el tiempo hasta la consolidación y la incidencia de complicaciones, pero la tasa de reoperaciones fue más alta en el grupo con banda de tensión, debido fundamentalmente a la extracción del material. Los resultados de nuestra serie indican que, si bien las tasas de consolidación ósea para la banda de tensión y el tornillo compresivo fueron altas $(94,2 \%$ y $85,7 \%$, respectivamente), la tasa de seudoartrosis en el grupo con tornillo compresivo fue del 14,2\%, esto representa un número casi tres veces superior a la tasa de la banda de tensión (5,7\%). No se observaron diferencias entre ambos grupos en el tiempo hasta lograr la consolidación ósea. En los pacientes con artrodesis y tornillo compresivo, la tasa de complicaciones fue cercana al $25 \%$ y todos los casos estuvieron relacionados con alteraciones en la consolidación ósea, esto contrasta con la incidencia de complicaciones en el grupo con banda de tensión $(11,4 \%)$. En nuestra serie, las reoperaciones se realizaron exclusivamente en los pacientes tratados con banda de tensión, en su mayoría, para extraer el material; sin embargo, no se puede tener certeza de la evolución y del pronóstico de los pacientes con artrodesis y tornillo compresivo que no consolidaron y que no fueron reoperados en el período de estudio.

Los resultados posoperatorios fueron satisfactorios y similares a los reportados por Breyer y cols. ${ }^{5}$ quienes llevaron a cabo el único estudio similar publicado hasta la fecha, según nuestro conocimiento. Ambos estudios cuentan con un número de pacientes y un seguimiento posoperatorio similares. En nuestra serie, la tasa de consolidación ósea fue del 94,2\% con banda de tensión y del 85,7\% con tornillo compresivo, cifras similares a las publicadas por Breyer y cols. ${ }^{5}$ (92,9\% y 87,5\%, respectivamente). En nuestro estudio, los tiempos promedio hasta la consolidación ósea fueron de 6.4 semanas en los pacientes con banda de tensión y de 7.2 semanas en aquellos con tornillo compresivo, valores más bajos que los de Breyer y cols..$^{5}$ (9.4 y 9.8 semanas, respectivamente). En nuestra serie, la incidencia de complicaciones fue del 11,4\% en el grupo con banda de tensión y del 23,8\% en el grupo con tornillo compresivo, tasas inferiores a las de Breyer y cols. ${ }^{5}$ (28,6\% con banda de tensión y 27,6\% con tornillo compresivo). No se obtuvo la consolidación ósea en cinco de 56 casos $(8,9 \%)$ y la tasa de seudoartrosis fue del $5,7 \%$ con la banda de tensión y del 14,2\% con el tornillo compresivo, estos valores coinciden con los publicados que informan un rango esperable de seudoartrosis del $0 \%$ al $15 \%$ en las artrodesis interfalángicas proximales y del $0 \%$ al $20 \%$ en las fusiones metacarpofalángicas del pulgar, ${ }^{27}$ y con los obtenidos por Breyer y cols.: $.^{5} 7,1 \%$ para la banda de tensión y 14,2\% para el tornillo compresivo. En nuestra serie, otra complicación fue una ruptura del tendón del extensor largo del pulgar a los 6 meses de la artrodesis metacarpofalángica del pulgar con banda de tensión. Stanley y cols. ${ }^{28}$ reportan esta complicación en dos casos de una serie de 42 artrodesis metacarpofalángicas del pulgar con clavijas de Kirschner. Seis de los 35 pacientes $(17,1 \%)$ con banda de tensión requirieron una nueva cirugía; ningún paciente del grupo con tornillo compresivo debió ser operado nuevamente. La intolerancia al material de osteosíntesis fue la causa de la reoperación en cuatro de los pacientes con banda de tensión (11,4\%). Las tasas de extracción del material de artrodesis con banda de tensión publicadas son: $9 \%$ en el estudio de Stern y cols., ${ }^{12}$ $13 \%$ en el de Uhl y Schneider, ${ }^{13} 26 \%$ en la serie de Khuri ${ }^{11}$ y $25 \%$ en el de Breyer y cols. ${ }^{5}$ La tasa de extracción del material en las series de artrodesis con tornillo compresivo varían del $0 \%$ al $4 \% .^{5,15,16,18}$

Por otro lado, de acuerdo con los resultados de esta serie, el tiempo de consolidación ósea fue menor en los pacientes no reumáticos, al margen de la técnica, mientras que, en el resto de los resultados posoperatorios, no se hallaron diferencias significativas entre pacientes reumáticos y no reumáticos.

Este estudio tiene ciertas limitaciones, principalmente su carácter retrospectivo, el número relativamente pequeño de casos y la selección no aleatoria de la técnica utilizada. Sin embargo, desde nuestra perspectiva, su fortaleza es que hay muy pocos estudios comparativos similares y que aquellos que comparan los resultados de estas dos técnicas entre pacientes reumáticos y no reumáticos son aún más escasos. 


\section{CONCLUSIONES}

Los resultados de este estudio comparativo indican que los métodos de banda de tensión y tornillo compresivo en las artrodesis interfalángica proximal y metacarpofalángica permiten lograr tasas altas de consolidación ósea. Sin embargo, debe tenerse en cuenta que la incidencia de seudoartrosis en los pacientes con tornillo compresivo fue prácticamente tres veces superior a la tasa de falta de consolidación en aquellos con banda de tensión. Por otro lado, no se observaron diferencias entre ambas técnicas en el tiempo hasta la consolidación ósea. Aproximadamente una cuarta parte de los casos con tornillo compresivo sufrió complicaciones y todas se relacionaron con alteraciones en la consolidación ósea, la cual fue significativamente más alta que la tasa de complicaciones del grupo con banda de tensión. Por otra parte, las reoperaciones se realizaron exclusivamente en los pacientes tratados con banda de tensión, sobre todo para extraer el material de osteosíntesis, pero de todas formas, no es posible tener certeza de la evolución y el pronóstico de los pacientes con artrodesis con tornillo compresivo que no consolidaron y que no fueron reoperados en el período de estudio.

Por último, el tiempo de consolidación ósea fue más corto en los pacientes no reumáticos, al margen de la técnica utilizada, mientras que, en el resto de los resultados posoperatorios, no se hallaron diferencias significativas entre los pacientes reumáticos y no reumáticos.

Conflicto de intereses: Los autores no declaran conflictos de intereses.

\section{BIBLIOGRAFÍA}

1. Jung J, Haghverdian B, Gupta R. Proximal interphalangeal joint fusion: indications and techniques. Hand Clin 2018;34(2):177-84. https://doi.org/10.1016/j.hcl.2017.12.007

2. Yamamoto M, Chung KC. Joint fusion and arthroplasty in the hand. Clin Plast Surg 2019;46(3):479-88. https://doi.org/10.1016/j.cps.2019.03.008

3. Sennwald G, Segmuller G. The metacarpo-phalangeal arthrodesis of the thumb according to the tension-band principle: indications and technique. Ann Chir Main 1983;2(1):38-45. https://doi.org/10.1016/s0753-9053(83)80078-7

4. Leibovic SJ. Proximal interphalangeal joint arthritis. En: Chhabra B, Isaacs J, eds. Arthritis and arthroplasty: The hand, wrist, and elbow. Philadelphia, PA: Elsevier Inc.; 2010:142-54.

5. Breyer JM, Vergara P, Parra L, Sotelo P, Bifani A, Andrade F. Metacarpophalangeal and interphalangeal joint arthrodesis: a comparative study between tension band and compression screw fixation. J Hand Surg Eur 2015;40(4):374-8. https://doi.org/10.1177/1753193413514362

6. Herren D. The proximal interphalangeal joint: arthritis and deformity. EFORT Open Rev 2019;4(6):254-62. https://doi.org/10.1302/2058-5241.4.180042

7. Uhl RL. Proximal interphalangeal joint arthrodesis using the tension band technique. J Hand Surg Am 2007;32(6): 914-7. https://doi.org/10.1016/j.jhsa.2007.04.014

8. Leibovic SJ. Instructional Course Lecture. Arthrodesis of the interphalangeal joints with headless compression screws. J Hand Surg Am 2007;32:1113-9. https://doi.org/10.1016/j.jhsa.2007.06.010

9. Hogh J, Jensen PO. Compression-arthrodesis of finger joints using Kirschner wires and cerclage. Hand 1982;14(2): 149-52. https://doi.org/10.1016/s0072-968x(82)80006-5

10. Hohendorff B, Franke J, Spies CK, Muller LP, Ries C. Arthrodesis of the proximal interphalangeal joint of fingers with tension band wire. Oper Orthop Traumatol 2017;29(5):385-94. https://doi.org/10.1007/s00064-016-0471-7

11. Khuri SM. Tension band arthrodesis in the hand. J Hand Surg Am 1986;11(1):41-5. https://doi.org/10.1016/s0363-5023(86)80099-5 
12. Stern PJ, Gates NT, Jones TB. Tension band arthrodesis of small joints in the hand. J Hand Surg Am 1993;18:194-7. https://doi.org/10.1016/0363-5023(93)90344-3

13. Uhl RL, Schneider LH. Tension band arthrodesis of finger joints: a retrospective review of 76 consecutive cases. J Hand Surg Am 1992;17:518-22. https://doi.org/10.1016/0363-5023(92)90365-v

14. Adla DN, Kitsis C, Miles AW. Compression forces generated by mini bone screws: a comparative study done on bone model. Injury 2005;36:65-70. https://doi.org/10.1016/j.injury.2004.03.026

15. Ayres JR, Goldstrohm GL, Miller GJ, Dell PC. Proximal interphalangeal joint arthrodesis with the Herbert screw. J Hand Surg Am 1988;13(4):600-3. https://doi.org/10.1016/s0363-5023(88)80105-9

16. Katzman SS, Gibeault JD, Dickson K, Thompson JD. Use of a Herbert screw for interphalangeal joint arthrodesis. Clin Orthop Relat Res 1993;(296):127-32. PMID: 8222414

17. Newman EA, Orbay MC, Nunez FA Jr, Nunez F. Minimally invasive proximal interphalangeal joint arthrodesis using headless screw: surgical technique. Tech Hand Surg 2018;22:39-42. https://doi.org/10.1097/BTH.0000000000000189

18. Teoh LC, Yeo SJ, Singh I. Interphalangeal joint arthrodesis with oblique placement of an AO lag screw. J Hand Surg Br 1994;19(2):208-11. https://doi.org/10.1016/0266-7681(94)90168-6

19. Capo JT, Melamed E, Shamian B, Hadley SR, Ng Lai W, Gerszberg K, et al. Biomechanical evaluation of 5 fixation devices for proximal interphalangeal joint arthrodesis. J Hand Surg Am 2014; 39(10):1971-7. https://doi.org/10.1016/j.jhsa.2014.07.035

20. Millrose M, Zach A, Kim S, Guthoff C, Eisenschenk A, Vonderlind HC. Biomechanical comparison of the proximal interphalangeal joint arthrodesis using a compression wire. Arch Orthop Trauma Surg 2019;139(4):577-81. https://doi.org/10.1007/s00402-019-03119-5

21. Vonderlind HC, Zach A, Eichenauer F, Kim S, Eisenschenk A, Millrose M. Proximal interphalangeal joint arthrodesis using a compression wire: A comparative biomechanical study. Hand Surg Rehabil 2019;38(5):307-11. https://doi.org/10.1016/j.hansur.2019.07.002

22. Alluri RK, Hill JR, Navo P, Ghiassi A, Stevanovic A, Mostofi A. Washer and post augmentation of 90/90 wiring for proximal interphalangeal joint arthrodesis: A biomechanical study. J Hand Surg Am 2018;43(12):1137. https://doi.org/10.1016/j.jhsa.2018.04.010

23. Mittelmeier W, Lehner S, Gollwitzer H, Hauschild M, Werber KD, Steinhauser E. Comparing biomechanical investigations about different wiring techniques of finger joint arthrodesis. Arch Orthop Trauma Surg 2005;125: 145-52. https://doi.org/10.1007/s00402-004-0773-3

24. Ijsselstein CB, van Egmond DB, Hovius SE, van der Meulen JC. Results of small-joint arthrodesis: comparison of Kirschner wire fixation with tension band wire technique. J Hand Surg Am 1992;17(5):952-6. https://doi.org/10.1016/0363-5023(92)90476-6

25. Leibovic SJ, Strickland JW. Arthrodesis of the proximal interphalangeal joint of the finger: comparison of the use of the Herbert screw with other fixation methods. J Hand Surg Am 1994;19(2):181-8. https://doi.org/10.1016/0363-5023(94)90002-7

26. Beldner S, Polatsch DB. Arthrodesis of the metacarpophalangeal and interphalangeal joints of the hand: Current concepts. J Am Acad Orthop Surg 2016;24(5):290-7. https://doi.org/10.5435/JAAOS-D-15-00033

27. Satteson ES, Langford MA, Li Z. The management of complications of small joint arthrodesis and arthroplasty. Hand Clin 2015;31(2):243-66. https://doi.org/10.1016/j.hcl.2015.01.002

28. Stanley JK, Smith EJ, Muirhead AG. Arthrodesis of the metacarpo-phalangeal joint of the thumb: a review of 42 cases. J Hand Surg Br 1989;14(3):291-3. https://doi.org/10.1016/0363-5023(94)90002-7 